\title{
Unilateral Purtscher-like retinopathy as the presenting feature of a case with spontaneous carotid artery dissection
}

\author{
Şefik Can İpek ${ }^{1}$, Aylin Yaman², Süleyman $\mathrm{Men}^{2}$, and Ali Osman Saatci ${ }^{2}$ \\ ${ }^{1}$ Affiliation not available \\ ${ }^{2}$ Dokuz Eylul Universitesi Tip Fakultesi
}

September 11, 2020

\begin{abstract}
Carotid artery dissection should be considered in the differential diagnosis when a clinician face with a case presenting with an unilateral Purtscher-like retinopathy.
\end{abstract}

Unilateral Purtscher-like retinopathy as the presenting feature of a case with spontaneous carotid artery dissection

Keywords: Dissection, internal carotid artery, Purtscher retinopathy.

\section{Key Clinical Message}

Carotid artery dissection should be considered in the differential diagnosis when a clinician face with a case presenting with an unilateral Purtscher-like retinopathy.

\section{Case description}

A 64-year-old man with type 2 diabetes was examined with a severe headache and mild visual acuity decrease in his right eye. On examination, his best-corrected visual acuity was $20 / 30$ in his right eye and 20/20 in the left. While left fundus was normal, there was peripapillary cotton-wool spots with an intact foveal architecture in his right eye (Figure 1). A diagnosis of right Purtscher-like retinopathy was made. A detailed systemic workup was carried out and right internal carotid artery catheter angiography demonstrated the presence of the spiral dissection of right internal carotid artery (Figure 2). The diagnosis of Purtscher-like retinopathy is clinical with a presentation that usually includes sudden unilateral or bilateral visual disturbance of variable severity. It is characterized with several retinal findings, such as cotton-wool spots, retinal hemorrhages, and areas of inner retinal whitening namely Purtscher flecken. A multitude of various systemic diseases has been described as the cause. ${ }^{1}$ Very recently Qazi et al. ${ }^{2}$ reported a 40 -year-old woman with an unilateral Purtscher-like retinopathy. Two days earlier, she had an ischemic stroke of her middle cerebral artery secondary to a right carotid artery dissection. Whenever a diagnosis of Purtscher-like retinopathy is made, underlying diseases should be scrutinized meticulously.

\section{Legends}

Figure 1- Right eye;

1. Color fundus picture showing the scattered peripapillary cotton-wool spots with notingly normal looking fovea.

2. Arteriovenous phase of fluorescein angiogram depicting the hypofluorescent spots corresponding to the cotton-wool spots. 
3. Horizontal spectral domain optical coherence tomographic frame through one of the cotton wool spots delineating the increased reflectivity of the ganglion cell layer.

Figure 2- Right internal carotid artery catheter angiography shows long segment dissection. The start and end points of the dissection are pointed by the arrows. As the dissected intima follows a spiral route, the true (flowing) lumen shows an undulating pattern.

\section{Author contribution:}

SCI- Involved in writing of the manuscript.

AY and AOS- Involved in the ophthalmological examination and editing of the manuscript.

SM- Involved in the neuroradiological examination.

Conflict of interest: None declared.

\section{References}

1. Miguel AIM, Henriques F, Azevedo LFR, Loureiro AJR, Maberley DAL. Systematic review of Purtscher's and Purtscher-like retinopathies.Eye . 2013;27(1):1-13.

2. Qazi S, Palakkamanil MM, Sia DIT, Ehmann DS. Carotid artery dissection associated with Purtscher-like retinopathy. Can J Ophthalmol . 2020;55(2):e77-e79.

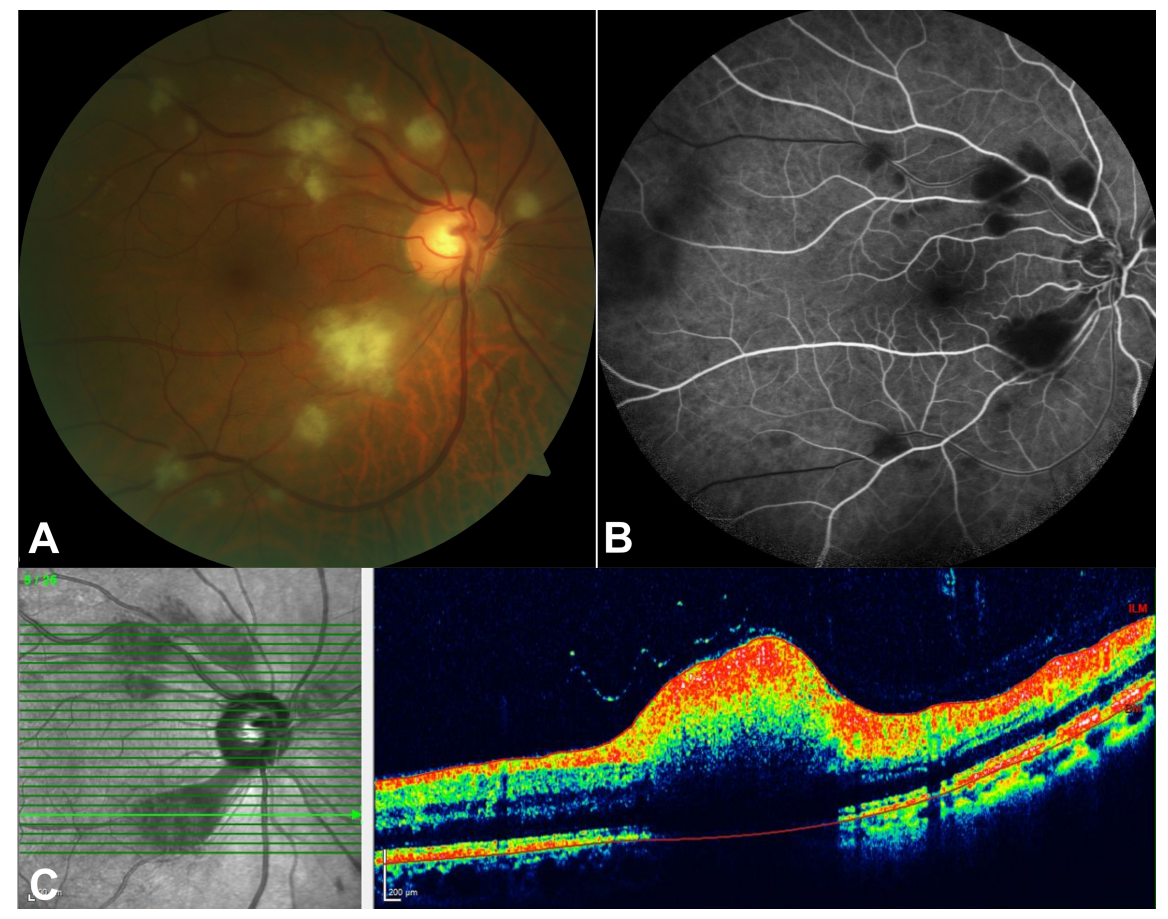




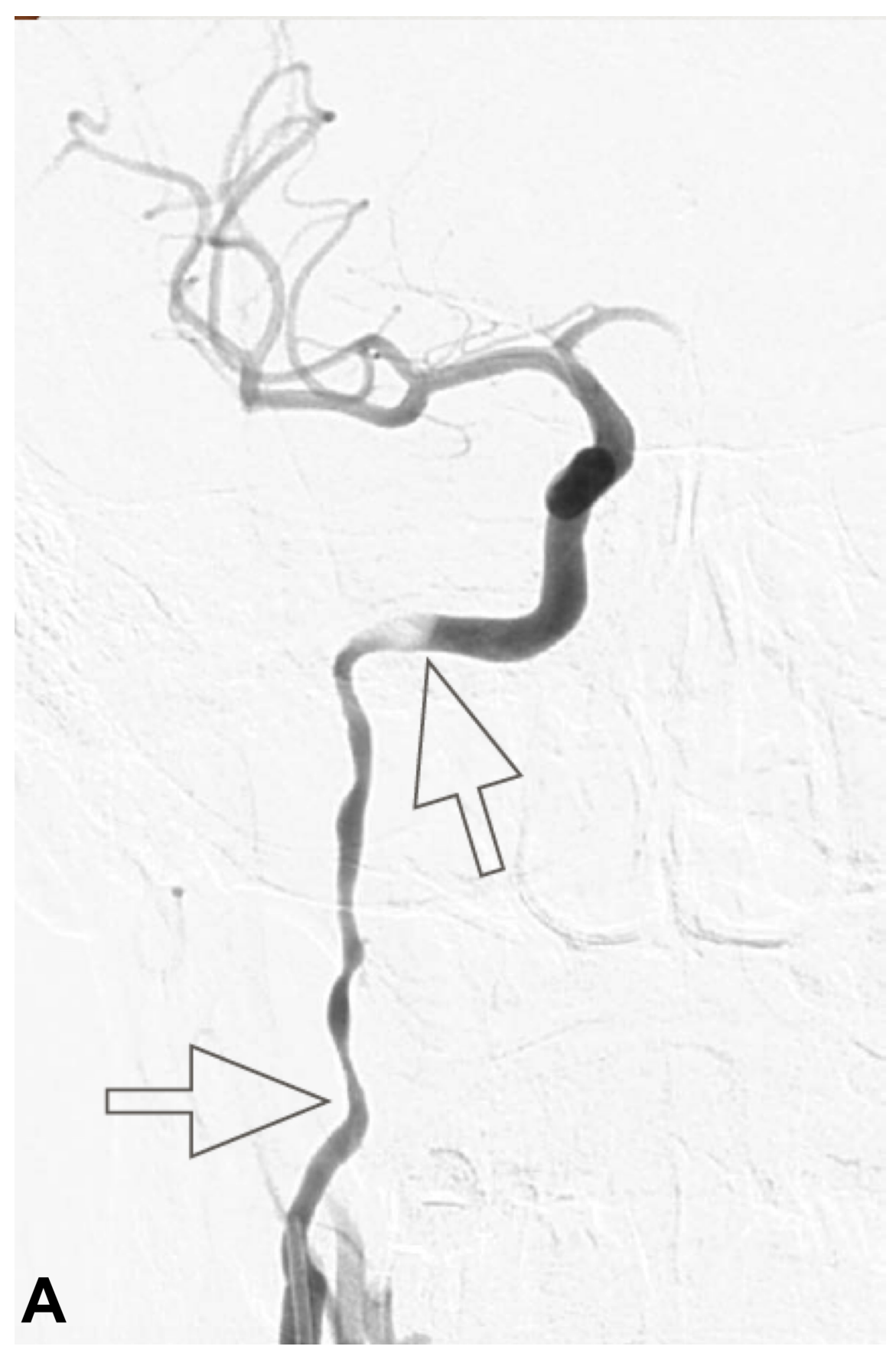

